\title{
Association of Traditional Chinese Medicine Therapy with Risk of Total Hip Replacement in Patients with Nontraumatic Osteonecrosis of the Femoral Head: A Population-Based Cohort Study
}

\author{
Yu-An Yeh, ${ }^{1}$ Jen-Huai Chiang, ${ }^{2,3}$ Mei-Yao Wu $\left(D^{4},{ }^{4}\right.$ Chun-Hao Tsai $\mathbb{D}^{5,},{ }^{3,5}$ \\ Horng-Chaung Hsu, ${ }^{3,5}$ Hsin-Cheng Hsu $\mathbb{D}^{1,6,7}$ and Tsung-Li Lin $\mathbb{D}^{5,8}$ \\ ${ }^{1}$ Chinese Traumatology, Department of Chinese Medicine, China Medical University Hospital, Taichung, Taiwan \\ ${ }^{2}$ Management Office for Health Data, China Medical University Hospital, Taichung, Taiwan \\ ${ }^{3}$ College of Medicine, China Medical University, Taichung, Taiwan \\ ${ }^{4}$ Chinese Internal Medicine, Department of Chinese Medicine, China Medical University Hospital, Taichung, Taiwan \\ ${ }^{5}$ Department of Orthopedics, China Medical University Hospital, Taichung, Taiwan \\ ${ }^{6}$ Graduate Institute of Clinical Medicine, China Medical University, Taichung, Taiwan \\ ${ }^{7}$ School of Post-Baccalaureate Chinese Medicine, College of Chinese Medicine, China Medical University, Taichung, Taiwan \\ ${ }^{8}$ Department of Sports Medicine, China Medical University, Taichung, Taiwan
}

Correspondence should be addressed to Tsung-Li Lin; alilin422@yahoo.com.tw

Received 12 September 2018; Revised 27 January 2019; Accepted 18 February 2019; Published 24 February 2019

Academic Editor: Takao Namiki

Copyright (C) 2019 Yu-An Yeh et al. This is an open access article distributed under the Creative Commons Attribution License, which permits unrestricted use, distribution, and reproduction in any medium, provided the original work is properly cited.

Background. Osteonecrosis of the femoral head (ONFH) contributes to $45 \%$ of total hip replacements (THRs) annually in Taiwan. Nontraumatic ONFH (NONFH) is multifactorial; no effective Western medicine is available to delay the disease process. This population-based cohort study investigated the association of traditional Chinese medicine (TCM) therapy with risk of THR in patients with NONFH. Methods. This retrospective study was conducted using claims data from all insured residents covered by the National Health Insurance from 2000 to 2010. We enrolled 1,680 newly diagnosed ONFH patients who had not undergone THR, before or within 6 months after diagnosis of ONFH; these patients did not exhibit hip fracture or dislocation before the endpoint. In total, 595 propensity score-matched pairs were selected from among 1,028 TCM users and 652 non-TCM users. The association between TCM use and risk of THR was analyzed using a Cox proportional hazard model. Kaplan-Meier and log rank tests were performed to plot the cumulative incidence of THR. Results. The mean follow-up periods were 5.00 years and 3.57 years for TCM and non-TCM cohorts, respectively. Compared to the non-TCM cohort, the TCM cohort had fewer patients undergoing THR surgery ( $25.4 \%$ vs. $18.2 \%$, adjusted hazard ratio: $0.60, p<0.0001)$. The risk of reduction was noted in the group aged $30-59$ years (adjusted hazard ratio: $0.56, p<0.0001$ ), but there was no association with gender nor socioeconomic status. There was a significantly lower cumulative incidence of THR in TCM users $(p<0.0001)$. Shu-Jing-Huo-Xue-Tang and Yan Hu Suo were the most frequently prescribed formula and single herb, respectively. Conclusions. NONFH patients using TCM had a lower risk of THR; the risk of reduction was noted in the group aged 30-59 years but was not associated with gender nor socioeconomic status. TCM might be useful in conservative treatment for NONFH.

\section{Introduction}

Osteonecrosis of the femoral head $(\mathrm{ONFH})$ is a major worldwide public health concern. Annually, there are 10,000 to 20,000 new cases reported in the United States [1] and
100,000 to 200,000 new cases reported in China [2]. The annual incidence of ONFH as an indication for total hip replacement (THR) has been reported as relatively low in the United States (10\%) [3] and the United Kingdom (2\%) [4]. Conversely, ONFH has been reported as a major indication 


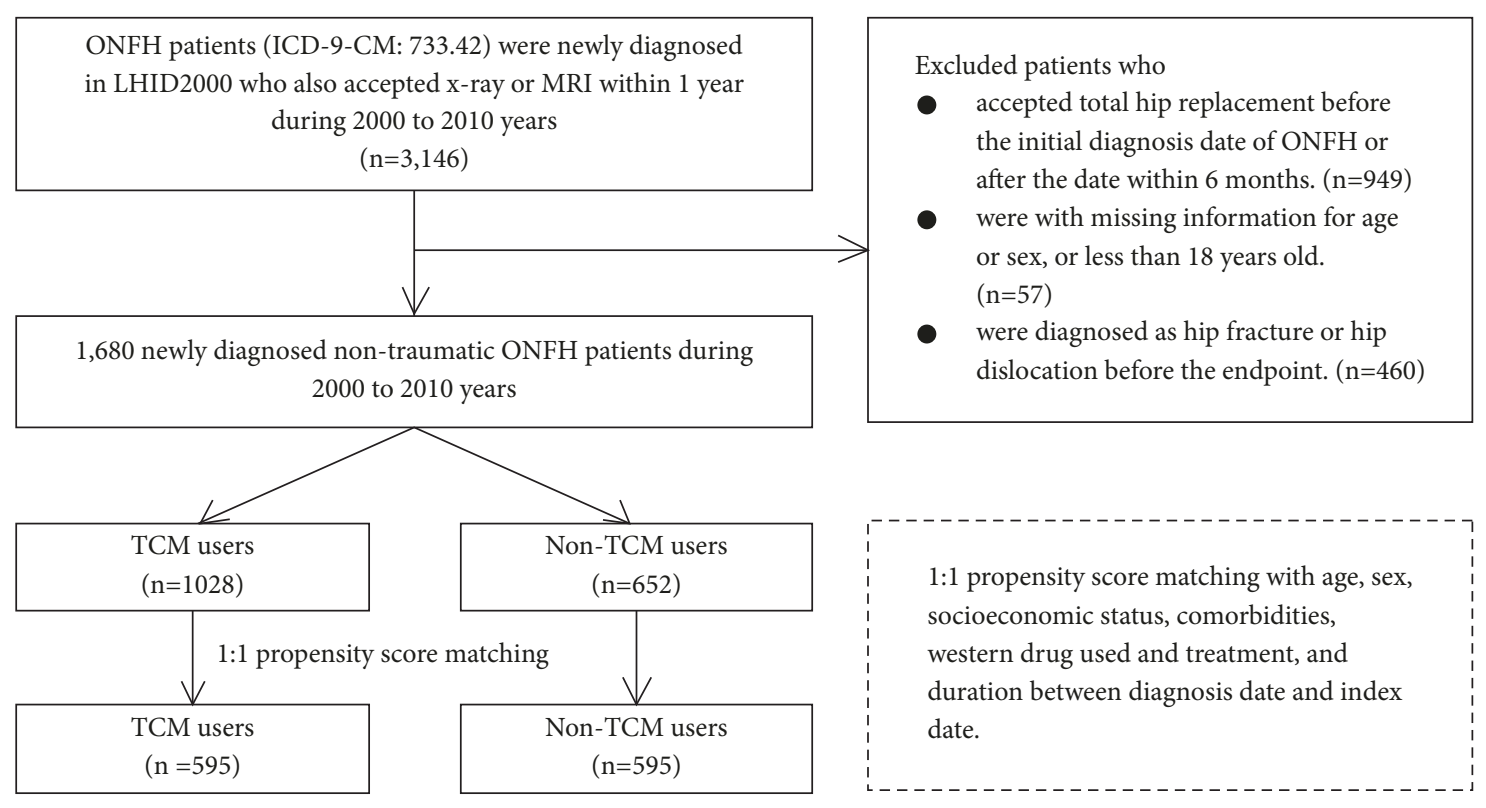

FIGURE 1: A flowchart about the selection of study subjects.

for THR in Asian populations: 50\% in Korea [5], 49\% in India [6], 45\% in Taiwan [7], 42\% in Singapore [8], and $41.2 \%$ in Hong Kong [9].

ONFH is defined as bone cell death due to interruption of the blood supply to the femoral head [10]. Typical ONFH occurs after physical trauma [11], whereas the etiology of nontraumatic ONFH (NONFH) is complex and multifactorial [12]. Steroid usage, alcohol consumption, autoimmune disease (e.g., systemic lupus erythematosus, vasculitis, and rheumatoid arthritis), diabetes, hyperlipidemia, renal failure, dialysis, pancreatitis, and hematologic diseases are all associated with increased risk of NONFH [12-16]. In Taiwan, alcohol consumption is the most prevalent etiology (45.2\%), followed by idiopathic causes (33.1\%) and steroid usage (21.7\%) [17].

Treatment of ONFH is stage-dependent [11]. Notably, many nonsurgical management strategies, such as Western pharmacological management strategies (lipid-lowering agents, anticoagulants, vasoactive substances, and bisphosphonates) and biophysical treatments (extracorporeal shockwave therapy [ESWT], pulse electromagnetic therapy [PET], and hyperbaric oxygen therapy [HBO]) have been found to improve pain and functional outcomes in the early stage [18]. However, most Western conservative therapies to prevent the onset of THR and delay its progression are controversial and lack sufficient evidence for widespread use [11, 18]. Surgical treatment, such as core or multiple drilling decompression in the early stage of $\mathrm{NONFH}$, may be effective in relieving symptoms; however, this approach has no greater value than conservative management in preventing collapse of the femoral head $[12,19,20]$.

Traditional Chinese medicine (TCM) has been widely applied in Asia and reportedly provides pain relief and improved quality of life in NONFH patients [21, 22]. However, no evidence has yet indicated that TCM could be useful in the prevention of THR. Therefore, the aim of this nationwide population-based retrospective cohort study was to investigate the association between use of TCM therapy and risk of THR in patients with NONFH.

\section{Materials and Methods}

2.1. Data Sources. In Taiwan, TCM has been covered under the government-run National Health Insurance (NHI) program since 1995 . This program ensures $>99 \%$ of citizens and is accepted by $>93 \%$ of healthcare institutes [23]. The NHI research database (NHIRD) provides ambulatory care, inpatient care, and management and medication data and is an ideal platform for use in pharmacoepidemiologic studies. Notably, it comprises a random sample that is representative of the general population; moreover, it includes registration files and original claims data for reimbursements, thereby avoiding selection bias and providing researchers with a comprehensive understanding of healthcare utilization, including Western medicine and TCM treatments $[24,25]$. All diseases in the NHIRD are classified in accordance with the International Classification of Diseases, Ninth Revision, and Clinical Modification (ICD-9-CM). For this study, we used the Longitudinal Health Insurance Database 2000 (LHID2000), which comprises all original claims data for 1 million individuals randomly selected from among all beneficiaries of the NHID.

2.2. Study Population. From January 1, 2000, to December 31,2010 , a total of 3,146 patients were newly diagnosed with ONFH (ICD-9-CM: 733.42) and underwent radiography or MRI within 1 year (Figure 1). We excluded patients who (1) underwent THR before the initial diagnosis of $\mathrm{ONFH}$ or within 6 months after the date of initial diagnosis $(n=949)$, (2) had records in which data were missing regarding age or sex, or who were less than 18 years of age $(n=57)$, and/or (3) 


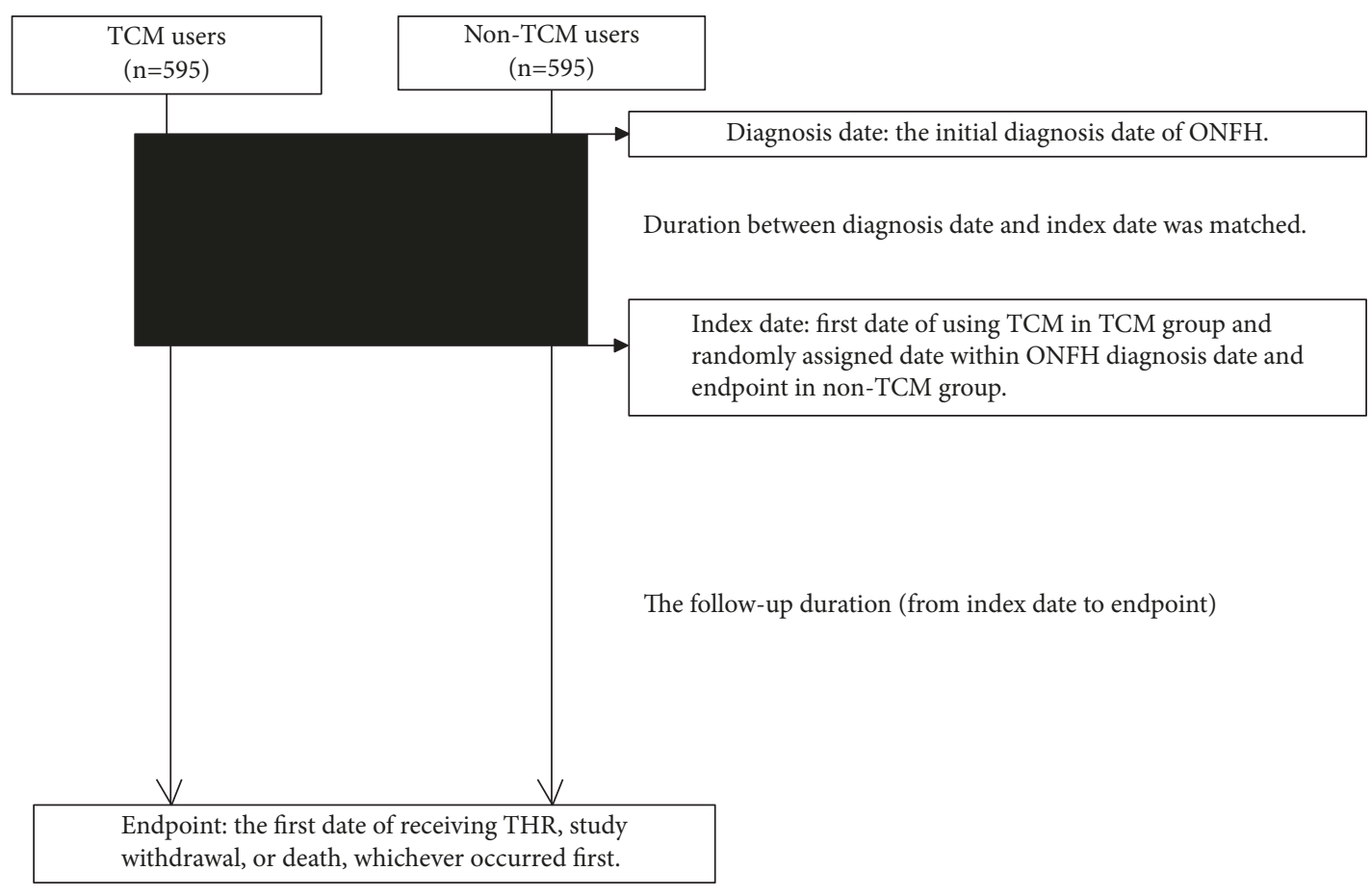

Figure 2: The definitions of diagnosis date, index date, and endpoint.

were diagnosed with hip fracture or hip dislocation (ICD-9CM: 733.14-15, 820.xx, 821.xx, 905.3, 718.25, 718.28-9, 718.3x, and $754.3 \mathrm{x}, 835 . \mathrm{xx})$ before the endpoint $(\mathrm{n}=460)$. Of the remaining 1,680 patients with $\mathrm{NONFH}$, those with at least one medical record in the TCM outpatient clinic were defined as TCM users $(n=1,028)$ and those with no TCM outpatient records were defined as non-TCM users $(n=652)$. For each TCM user, a control subject who was a non-TCM user was randomly selected by propensity score matching, according to age, sex, socioeconomic status, comorbidities, Western drugs used, surgical treatments, and the duration between the diagnosis date and the index date. Diagnosis date was the initial date of NONFH diagnosis. Index date was the first date of TCM use in the TCM group, whereas it was a randomly assigned date between the diagnosis date and the endpoint (see "Primary Outcome" subsection for details) in the nonTCM group (Figure 2). Finally, 595 propensity-matched pairs of TCM and non-TCM patients were used in this analysis.

2.3. Primary Outcome. The primary outcome was THR during the study period (2000 to 2013). The endpoint was the date of THR, study withdrawal, or death, whichever occurred first (Figure 2).

2.4. Covariate Assessment. Sociodemographic factors assessed in this study included age, sex, and socioeconomic status. Age was divided into three groups: 18-29 years, $30-59$ years, and $\geq 60$ years. Sex was categorized as male or female. Socioeconomic status was divided into two groups according to monthly salary: fixed premium and dependent $(<20,000$ New Taiwan Dollar, NTD; 1 USD = 30 NTD $)$ and ensured salary grading above fixed premium $(\geq 20,000$
NTD). Baseline comorbidities were considered present if the following ICD-9-CM codes appeared in at least three outpatient claims or one inpatient claim before the initial date of ONFH diagnosis: alcohol-related disease (ICD-9-CM: 291, 303, 303.0, 303.00-03, 303.9, 303.90-93, 305.0, 305.01-03, 357.5, 425.5, 535.3, 535.30-31, 571.0-3, 790.3, 977.3, 980.0, and 980.8-9); autoimmune disease: lupus erythematosus (ICD-9-CM: 695.4, 373.34, 710.0), vasculitis (ICD-9-CM: 136.1, 443.1, 446.0-2, 446.4-5, and 446.7), and rheumatoid arthritis (ICD-9-CM: 714.0); diabetes (ICD-9-CM: 250), hyperlipidemia (ICD-9-CM: 272.0-5, 272.7-9); pancreatitis (ICD-9-CM: 577.0-1); hematologic disease (ICD-9-CM: 205, 238.4, 282, 284, and 286); and renal failure or dialysis (ICD-9-CM: 584-586, 403.01, 403.11, 403.91, 404.02, 404.12, and 404.92; treatment code: 58001C, 58027C, 58029C, and 58002C). Western medicines (e.g., bisphosphonate, anticoagulants, iloprost, statins, oral steroids, or intravenous steroids) and surgical treatments (e.g., cord decompression, bone graft, and osteotomy) between initial diagnosis date and endpoint were also considered.

2.5. Statistical Analyses. Differences between the two groups were assessed using the chi-squared test or Fisher's exact test for categorical variables and by Student's t-test for continuous variables. The Cox proportional hazard model with hazard ratios (HR) and 95\% confidence intervals (CI) was used to estimate the association between TCM use and the risk of THR, as well as the risks between TCM use and each of multiple covariates among NONFH patients. KaplanMeier analysis and log rank tests were performed to plot the cumulative incidence of THR. A $p$ value $<0.05$ was considered to be statistically significant. All analyses were 
TABLE 1: Characteristics of NONFH patients according to use of TCM.

\begin{tabular}{|c|c|c|c|c|c|}
\hline \multirow{3}{*}{ Variables } & \multicolumn{4}{|c|}{ TCM } & \multirow[t]{3}{*}{$p$-value $*$} \\
\hline & \multicolumn{2}{|c|}{ No $(n=595)$} & \multicolumn{2}{|c|}{ Yes $(n=595)$} & \\
\hline & $\mathrm{n}$ & $\%$ & $\mathrm{n}$ & $\%$ & \\
\hline $\operatorname{Sex}$ & & & & & 0.8437 \\
\hline Female & 159 & 26.72 & 156 & 26.22 & \\
\hline Male & 436 & 73.28 & 439 & 73.78 & \\
\hline Age group & & & & & 0.8857 \\
\hline $18-29$ & 34 & 5.71 & 38 & 6.39 & \\
\hline $30-59$ & 390 & 65.55 & 386 & 64.87 & \\
\hline$\geq 60$ & 171 & 28.74 & 171 & 28.74 & \\
\hline Mean \pm SD & & & & & $0.9124^{\mathrm{a}}$ \\
\hline Socioeconomic status & & & & & 0.5815 \\
\hline Fixed premium and dependent & 359 & 60.34 & 348 & 58.49 & \\
\hline Insured salary grading above fixed premium & 236 & 39.66 & 247 & 41.51 & \\
\hline \multicolumn{6}{|l|}{ Comorbidity } \\
\hline Alcohol-related disease & 387 & 65.04 & 384 & 64.54 & 0.8555 \\
\hline Autoimmune disease & 16 & 2.69 & 32 & 5.38 & 0.0184 \\
\hline Diabetes & 107 & 17.98 & 118 & 19.83 & 0.4154 \\
\hline Hyperlipidemia & 148 & 24.87 & 153 & 25.71 & 0.7388 \\
\hline Pregnancy & 14 & 2.35 & 24 & 4.03 & 0.0992 \\
\hline Renal failure or dialysis & 47 & 7.9 & 42 & 7.06 & 0.5816 \\
\hline Pancreatitis & 19 & 3.19 & 13 & 2.18 & 0.2823 \\
\hline Hematologic disease & 9 & 1.51 & 5 & 0.84 & 0.2822 \\
\hline \multicolumn{6}{|l|}{ Drug used } \\
\hline Bisphosphonate & 24 & 4.03 & 19 & 3.19 & 0.4374 \\
\hline Anticoagulants & 103 & 17.31 & 112 & 18.82 & 0.4977 \\
\hline Iloprost & 0 & 0 & 0 & 0 & - \\
\hline Statins & 156 & 26.22 & 165 & 27.73 & 0.5566 \\
\hline Oral Steroid + IV Steroid & 538 & 90.42 & 540 & 90.76 & 0.8426 \\
\hline \multicolumn{6}{|l|}{ Treatment } \\
\hline Cord decompression & 38 & 6.39 & 48 & 8.07 & 0.2629 \\
\hline Bone graft (BG) & 16 & 2.69 & 28 & 4.71 & 0.0653 \\
\hline Osteotomy & 1 & 0.17 & 1 & 0.17 & $1.0000^{\mathrm{b}}$ \\
\hline $\begin{array}{l}\text { Duration between diagnosis date and index date } \\
\text { (days) (mean, median) }\end{array}$ & \multicolumn{2}{|c|}{$678(355)$} & \multicolumn{2}{|c|}{$699(383)$} & $0.6528^{a}$ \\
\hline
\end{tabular}

Abbreviations. NONFH: nontraumatic osteonecrosis of the femoral head; TCM, traditional Chinese medicine. $*$ Chi-squared test; ${ }^{\text {a }}$ t-test; ${ }^{\mathrm{b}}$ Fisher's exact test. The mean (median) of the follow-up period was 5.00 (4.38) years and 3.57 (3.35) years for the TCM user cohort and non-TCM user cohort, respectively.

performed using SAS statistical software (version 9.4 for Windows; SAS Institute, Inc., Cary, NC, USA).

2.6. Ethical Consideration. All patient information that could be used to identify individuals or care providers was deidentified and encrypted before release. The study was approved by the research ethics committee of China Medical University and Hospital (CMUH104-REC2-115(CR-2)).

\section{Results}

There were no statistically significant differences in age, sex, socioeconomic status, most comorbidities (except autoimmune disease), Western drug use, and surgical treatments between the non-TCM and TCM groups (Table 1). Most patients were male in both groups $(73.28 \%$ vs $73.78 \%$ ). The 30-59-year-old group comprised the largest proportion in both groups (65.55\% vs. $64.87 \%)$. Nearly $60 \%$ of patients had income $<20,000$ NTD per month (fixed premium and dependent group). Alcohol-related disease was the most common comorbidity, followed by hyperlipidemia and diabetes. Before the endpoint, $90.42 \%$ of non-TCM users and $90.76 \%$ of TCM users had used oral or intravenous steroids. The mean (median) follow-up periods (from index date to endpoint) were 5.00 (4.38) years and 3.57 (3.35) years for the TCM and non-TCM cohorts. 


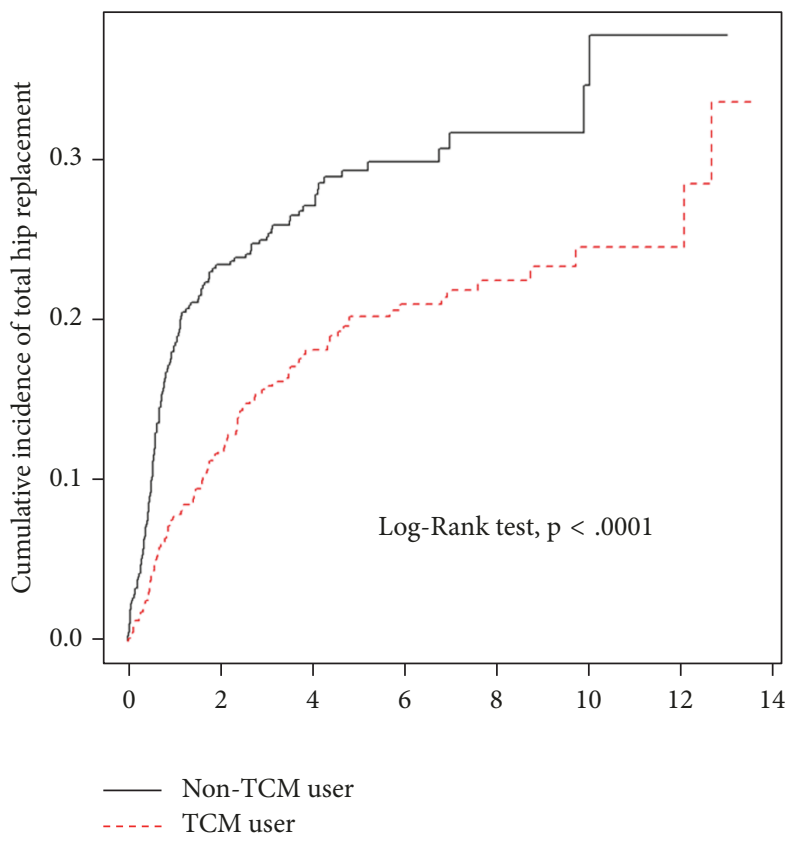

FIgURE 3: Kaplan-Meier curves of cumulative incidence of THR in patients with NONFH according to TCM use.

In the TCM cohort, fewer patients underwent THR surgery, compared to the non-TCM cohort (18.2\% vs $25.4 \%)$; moreover, the TCM cohort had a lower adjusted hazard ratio (0.60, 95\% CI: 0.46-0.77, $p<0.0001)$, after adjusting for age, sex, socioeconomic status, comorbidities, Western drug use, and surgical treatments (Table 2). Males had a lower risk of THR than females. However, age and socioeconomic status did not affect the risk of THR.

The TCM cohort, compared to the non-TCM cohort, had a significantly lower incidence rate of THR for women and men (Table 3 ). The TCM cohort also had a significantly lower incidence rate in the 30-59-year-old subgroup. Although there were more THR events in the $\geq 60$-year-old subgroup in the TCM group, the adjusted hazard ratio was equal to $1(95 \%$ CI: 0.58-1.72). However, the TCM cohort had fewer patients undergoing THR surgery regardless of socioeconomic status.

Furthermore, Kaplan-Meier analysis with a log rank test showed a significantly lower cumulative incidence of THR in the TCM cohort $(p<0.0001)$ than in the non-TCM cohort; the cumulative incidence rate in the TCM cohort remained lower for a follow-up period of up to 12 years (Figure 3 ).

Table 4 shows the 10 most frequently prescribed formulae and single herbs for treating NONFH in Taiwan. Among multiherb products, Shu-Jing-Huo-Xue-Tang (4,106 persondays) was the most commonly prescribed formula. The herb Yan Hu Suo (4,348 person-days), produced by extraction of a single substance, was the most frequently prescribed single herb.

\section{Discussion}

This study is the first population-based retrospective cohort study regarding the association between TCM use and risk of
THR in NONFH patients in Taiwan. Our principal findings were as follows: TCM users had a 0.60 -fold lower risk of THR. The risk of reduction was noted in the group aged 30-59 years but was not associated with gender nor socioeconomic status. Furthermore, there was a significantly lower cumulative incidence of THR in TCM users.

TCM has been shown to raise Harris functional scores and improve the quality of life among NONFH patients [21, 22]. However, there has been no large-scale study regarding the potential of TCM to lower the risk of requiring joint replacement surgery. In our study, we enrolled 1,120 NONFH patients, evenly divided into TCM users and nonusers; these patients were matched on the basis of age, sex, socioeconomic status, drugs (including steroids) used, and other treatments that may affect the progression of the disease $[12,17]$. We found that male sex and alcohol consumption were prevalent among NONFH patients (Table 1). These results are similar to findings from other studies in Taiwan [17]. The 30-59-yearold age group included $65 \%$ of the patients, which was consistent with a previous finding that NONFH occurs most often between the ages of 30 and 50 [26]. We found that nearly $60 \%$ of patients with NONFH were fixed premium and dependent group. Because a previous study showed that low-income patients were less likely than their high-income counterparts to undergo total THR [27], we matched the patients on the basis of socioeconomic status (Table 1) and adjusted the analyses based on income (Table 2). These adjusted results show that TCM users had a significantly lower risk of THR, regardless of socioeconomic status (Table 3). Furthermore, TCM users in all age groups had lower incidence rates of THR. However, only in the $30-59$-year-old group (65\% of patients) had significantly lower risk. The result may be due to the small sample size among patients aged 18-29 and aged $\geq 60$. 


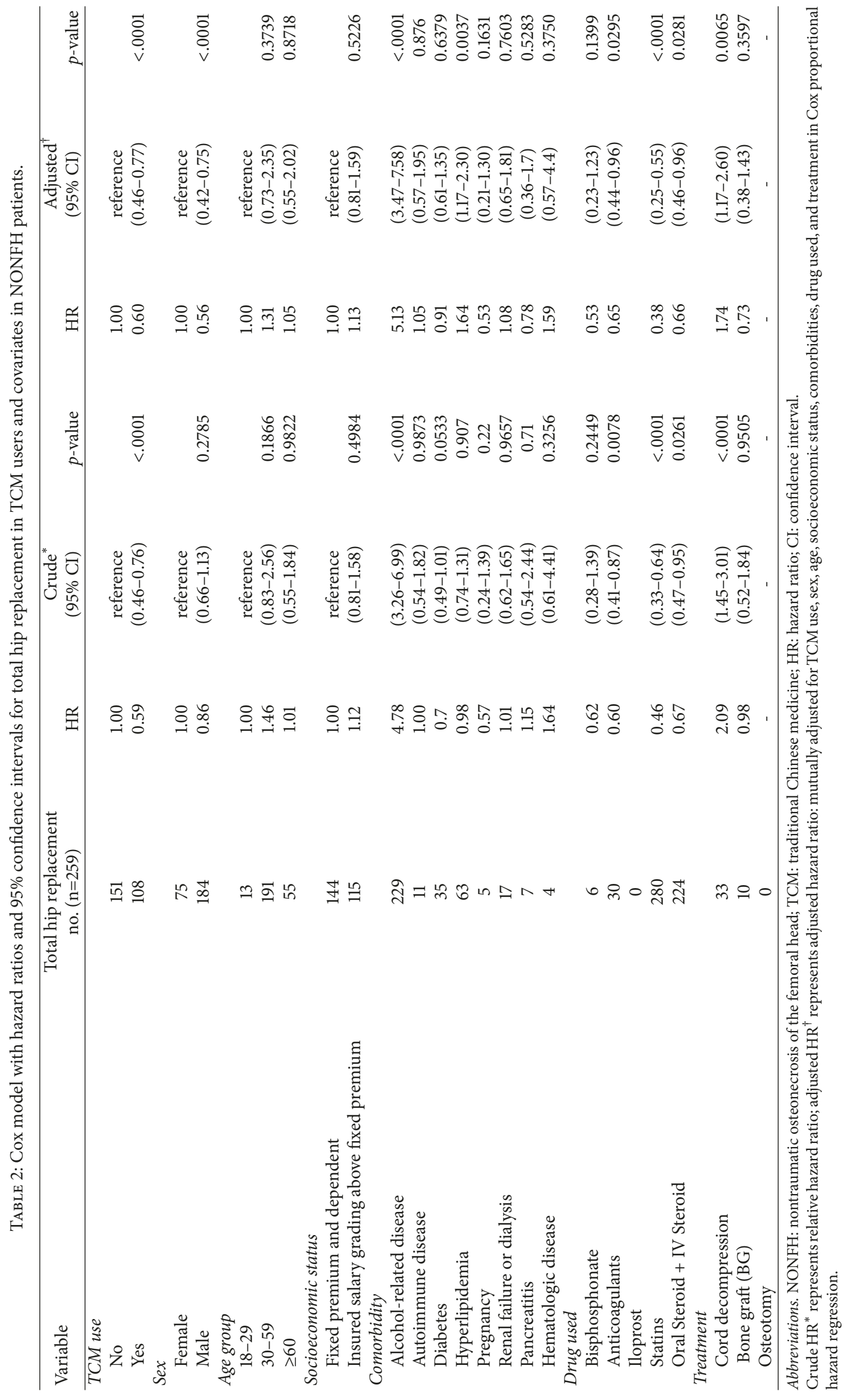




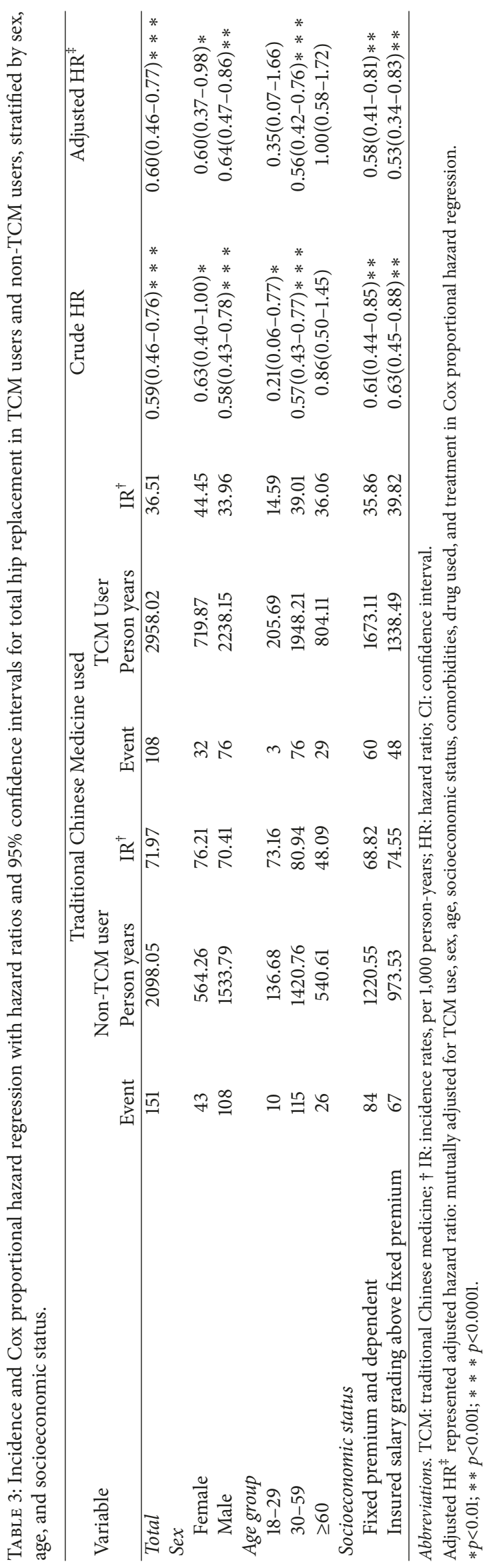




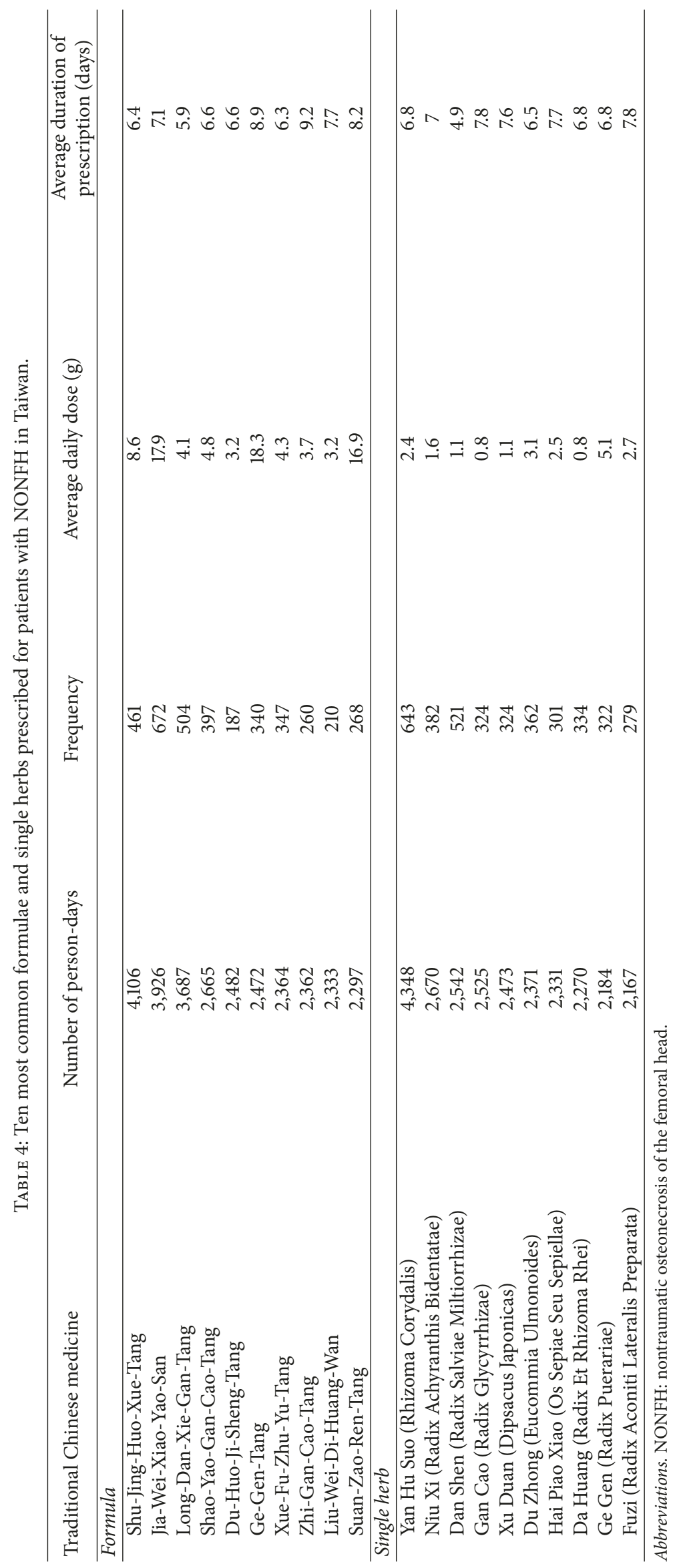


NONFH appears to be related to hypercoagulable states, suppression of angiogenesis, hyperadipogenesis, genetic factors, and transition from bone remodeling to bone resorption [16]. Various immune mechanisms and activation of proinflammatory pathways have also been associated with NONFH [12]. The mechanism by which TCM affects $\mathrm{NONFH}$ is unclear and may vary on the basis of herbs or formulae used. TCM herb extractions or formulae can reportedly counteract endothelial injury, excessive adipogenesis, blood stasis, and apoptosis; moreover, they can improve osteogenesis, angiogenesis, and blood circulation, both in vitro and in vivo [28-30]. In the present study, the 10 most commonly prescribed formulae and single herbs were as follows. Shu-Jing-Huo-Xue-Tang (SJHXT) was the most commonly prescribed formulae; this has been shown to relieve muscle pain [31], increase blood circulation [32], and enhance antioxidant enzymatic activity [33]. SJHXT also exhibits anti-inflammatory and analgesic activity $[34,35]$. JiaWei-Xiao-Yao-San (JWXYS) and Long-Dan-Xie-Gan-Tang (LDXGT) mediate anti-inflammatory activity through inhibition of cytokines and proinflammatory enzymes [36, 37]. However, they have not been shown to improve NONFH. Shao-Yao-Gan-Cao-Tang (SYGCT) has been reported to relieve muscle spasm [38] and spasmodic pain in the musculoskeletal system, including joints, back, and soft tissues $[39,40]$. SJHXT and SYGCT can enhance kinase signal pathways, which are important in osteoblast differentiation $[41,42]$. Du-Huo-Ji-Sheng-Tang (DHJST) can regulate the expression of vascular endothelial growth factor and hypoxiainducible factor, inhibit chondrocyte apoptosis [43], promote osteogenic differentiation, and decrease the aging process of human mesenchymal stem cells [44]. These characteristics may be related to the improvement of NONFH. Ge-GenTang (GGT) is named for its major herb composition, GeGen, which was also a commonly prescribed single herb in our cohort study. The major active compound of GeGen, puerarin, reportedly inhibits adipogenic differentiation and prevents alcohol-induced osteonecrosis [29]. Xue-FuZhu-Yu-Tang (XFZYT) is used to enhance blood circulation, counteract blood stasis, and inhibit inflammatory responses and apoptosis $[45,46]$.

Among single herbs, Yan Hu Suo (YHS) was prescribed most frequently. Tetrahydroprotoberberines, dehydrocorybulbine, and L-tetrahydropalmatine are active components that can be isolated from YHS; these have been demonstrated to exert analgesic and antinociceptive effects on chronic inflammatory and injury-induced neuropathic pain [47-50]. $\mathrm{Niu} \mathrm{Xi}(\mathrm{NX})$ has been reported to induce angiogenesis and is used as treatment for bone injury [51]; it is a key component in both SJHXT and XFZYT and was commonly used as treatment for NONFH in our study. Moreover, NX is a key component of the Huogu II formula, which showed preventive and therapeutic effects in an experimental model of ONFH [52]. Dan Shen (DS) has lipid-lowering [53] and anti-inflammatory [54] effects; furthermore, it could protect endothelial cells from hydrogen peroxide damage and inhibit apoptosis [55]. Gan Cao (GC) is very popular in TCM and commonly used in herbal formulae to "harmonize" other ingredients; it has anti-inflammatory, antioxidative, and immunomodulatory effects $[56,57]$.

Each Chinese herb or formula might have different osteogenic, angiogenic, anti-inflammatory, antioxidant, antiadipogenic, and analgesic effects, which contribute to the effect of TCM treatment in reducing the risk of THR among NONFH patients. Overall, TCM treatment might be protective for patients with NONFH.

\section{Limitations}

This study had some limitations. First, we did not evaluate the amount and frequency of steroid or alcohol usage. Second, Western treatments, such as ESWT, PET, HBO, tantalum implants, and biological agents that lack sufficient evidence, were not covered by the NHI program; thus, these were not evaluated. Herbal decoction and folk medicine are also not covered by the NHI program. Therefore, the utilization of TCM may be underestimated. Third, NONFH severity and stage were not assessed. However, we excluded patients who underwent THR before the initial diagnosis date, as well as those who underwent THR within 6 months after the initial diagnosis date. Finally, we did not assess any adverse drug reactions or interactions between Chinese medicine and Western medicine, although these have been previously reported. Despite these limitations, our findings are representative of the general population because we used population-based data from a national database.

\section{Conclusions}

In this retrospective population-based cohort study, TCM users had a lower risk of THR. The risk of reduction was noted in the group aged 30-59 years but was not associated with gender nor socioeconomic status. We also found a significantly lower cumulative incidence of THR among TCM users. However, further prospective studies examining the mechanisms of TCM are needed to confirm our findings.

\section{Data Availability}

The data used to support the findings of this study are included within the article.

\section{Disclosure}

The authors and their families have no commercial associations or sources of support. No benefits in any form have been or will be received from a commercial party related directly or indirectly to the subject of this manuscript.

\section{Conflicts of Interest}

The authors declare that they have no conflicts of interest.

\section{Authors' Contributions}

Hsin-Cheng Hsu and Tsung-Li Lin contributed equally to this work. 


\section{Acknowledgments}

This study is supported in part by Taiwan Ministry of Health and Welfare Clinical Trial Center (MOHW108-TDUB-212-133004), China Medical University Hospital, Academia Sinica Stroke Biosignature Project (BM10701010021), MOST Clinical Trial Consortium for Stroke (MOST 107-2321-B-039 -004-), Tseng-Lien Lin Foundation, Taichung, Taiwan, and Katsuzo and Kiyo Aoshima Memorial Funds, Japan.

\section{References}

[1] M. A. Mont, M. G. Zywiel, D. R. Marker, M. S. McGrath, and R. E. Delanois, "The natural history of untreated asymptomatic osteonecrosis of the femoral head: a systematic literature review," The Journal of Bone \& Joint Surgery, vol. 92, no. 12, pp. 2165-2170, 2010.

[2] F. Liu, W. Wang, L. Yang et al., "An epidemiological study of etiology and clinical characteristics in patients with nontraumatic osteonecrosis of the femoral head," Journal of Research in Medical Sciences, vol. 22, no. 1, p. 15, 2017.

[3] M. A. Mont and D. S. Hungerford, "Non-traumatic avascular necrosis of the femoral head," The Journal of Bone \& Joint Surgery, vol. 77, no. 3, pp. 459-474, 1995.

[4] National Joint Registry for England WaNI, "NJR 10th Annual Report 2013," 2013, http://www.njrcentre.org.uk.

[5] J.-D. Chang, M. Hur, S.-S. Lee, J.-H. Yoo, and K. M. Lee, "Genetic background of nontraumatic osteonecrosis of the femoral head in the Korean population," Clinical Orthopaedics and Related Research, vol. 466, no. 5, pp. 1041-1046, 2008.

[6] J. Pachore, S. Vaidya, C. Thakkar, H. K. Bhalodia, and H. Wakankar, "ISHKS joint registry: a preliminary report," Indian Journal of Orthopaedics, vol. 47, no. 5, pp. 505-509, 2013.

[7] K.-A. Lai, W.-J. Shen, C.-Y. Yang, C.-J. Shao, J.-T. Hsu, and R.M. Lin, "The use of alendronate to prevent early collapse of the femoral head in patients with nontraumatic osteonecrosis: a randomized clinical study," The Journal of Bone \& Joint Surgery, vol. 87, no. 10, pp. 2155-2159, 2005.

[8] G. Singh, L. Krishna, and S. D. De, "The ten-year pattern of hip diseases in Singapore," Journal of Orthopaedic Surgery (Hong Kong), vol. 18, no. 3, pp. 276-278, 2010.

[9] V. W. Chan, P. Chan, K. Chiu, C. Yan, and F. Ng, "Why do Hong Kong patients need total hip arthroplasty? An analysis of 512 hips from 1998 to 2010," Hong Kong Medical Journal, vol. 22, no. 1, pp. 11-15, 2016.

[10] Y. Assouline-Dayan, C. Chang, A. Greenspan, Y. Shoenfeld, and M. E. Gershwin, "Pathogenesis and natural history of osteonecrosis," Seminars in Arthritis and Rheumatism, vol. 32, no. 2, pp. 94-124, 2002.

[11] J. Moya-Angeler, A. L. Gianakos, J. C. Villa, A. Ni, and J. M. Lane, "Current concepts on osteonecrosis of the femoral head," World Journal of Orthopedics, vol. 6, no. 8, pp. 590-601, 2015.

[12] M. A. Mont, J. J. Cherian, R. J. Sierra, L. C. Jones, and J. R. Lieberman, "Nontraumatic osteonecrosis of the femoral head: Where dowe stand today? A ten-year update," Journal of Bone and Joint Surgery - American Volume, vol. 97, no. 19, pp. 16041627, 2014.

[13] T. Shigemura, J. Nakamura, S. Kishida et al., "Incidence of osteonecrosis associated with corticosteroid therapy among different underlying diseases: prospective MRI study," Rheumatology, vol. 50, no. 11, pp. 2023-2028, 2011.
[14] X.-S. Wang, Q.-Y. Zhuang, X.-S. Weng, J. Lin, J. Jin, and W.-W. Qian, "Etiological and clinical analysis of osteonecrosis of the femoral head in Chinese patients," Chinese Medical Journal, vol. 126, no. 2, pp. 290-295, 2013.

[15] L. Chokotho, W. J. Harrison, N. Lubega, and N. C. Mkandawire, "Avascular necrosis of the femoral head in HIV positive patients-an assessment of risk factors and early response to surgical treatment," Malawi Medical Journal, vol. 25, no. 2, pp. 28-32, 2013.

[16] F. Pouya and M. A. Kerachian, "Avascular necrosis of the femoral head: Are any genes involved?" Archives of Bone and Joint Surgery, vol. 3, no. 3, pp. 149-155, 2015.

[17] S. Tsai, P. Wu, C. Chen et al., "Etiologies and outcome of osteonecrosis of the femoral head: etiology and outcome study in a taiwan population," Journal of the Chinese Medical Association, vol. 79, no. 1, pp. 39-45, 2016.

[18] M. Chughtai, N. S. Piuzzi, A. Khlopas, L. C. Jones, S. B. Goodman, and M. A. Mont, "An evidence-based guide to the treatment of osteonecrosis of the femoral head," The Bone \& Joint Journal, vol. 99-B, no. 10, pp. 1267-1279, 2017.

[19] K. Koo, R. Kim, G. Ko, H. Song, S. Jeong, and S. Cho, "Preventing collapse in early osteonecrosis of the femoral head. A randomised clinical trial of core decompression," The Journal of Bone \& Joint Surgery (British Volume), vol. 77-B, no. 6, pp. 870-874, 1995.

[20] J. G. Wright, T. A. Einhorn, and J. D. Heckman, "Grades of recommendation," The Journal of Bone \& Joint Surgery, vol. 87, no. 9, pp. 1909-1910, 2005.

[21] W. Wang, J. Yu, and X. Tao, "Systemic lupus erythematosus complicated with femoral head ischemic necrosis treated by Chinese medicine therapy for activating blood and dredging collaterals method," Chinese Journal of Integrative Medicine, vol. 17, no. 2, pp. 105-110, 2011.

[22] C.-G. Li, L. Shen, Y.-P. Yang, X.-J. Xu, B. Shuai, and C. Ma, "Effects of Modified Qinge Pill () on the expression of adiponectin, bone morphogenetic protein 2 and coagulationrelated factors in patients with nontraumatic osteonecrosis of the femoral head," Chinese Journal of Integrative Medicine, vol. 23, no. 3, pp. 183-189, 2017.

[23] NHIA, National health insurance annual report 2015-2016, National Health Insurance Administration, Ministry of Health and Welfare, Taipei, Taiwan, 2016.

[24] Y.-C. Lee, Y.-T. Huang, Y.-W. Tsai et al., “The impact of universal National Health Insurance on population health: the experience of Taiwan," BMC Health Services Research, vol. 10, article 225, 2010.

[25] A. W. Hsing and J. P. A. Ioannidis, "Nationwide population science: lessons from the Taiwan national health insurance research database," JAMA Internal Medicine, vol. 175, no. 9, pp. 1527-1529, 2015.

[26] S. Dempsey and H. Michael, “The adult hip," in Turek's Orthopaedics: Principles and their Application, S. L. Weinstein and J. A. Buckwalter, Eds., vol. 533, LIppincott Williams \& Wilkins, Philadelphia, Pa, USA, 6th edition, 2008.

[27] N. Agabiti, S. Picciotto, G. Cesaroni et al., "The influence of socioeconomic status on utilization and outcomes of elective total hip replacement: a multicity population-based longitudinal study," International Journal for Quality in Health Care, vol. 19, no. 1, pp. 37-44, 2007.

[28] G. Zhang, X. Wang, H. Sheng et al., "Constitutional flavonoids derived from epimedium dose-dependently reduce incidence 
of steroid-associated osteonecrosis not via direct action by themselves on potential cellular Targets," PLOS ONE, vol. 4, no. 7, p. e6419, 2009.

[29] Y. Wang, L. Yin, Y. Li, P. Liu, and Q. Cui, "Preventive effects of puerarin on alcohol-induced osteonecrosis," Clinical Orthopaedics and Related Research, vol. 466, no. 5, pp. 10591067, 2008.

[30] X. Wang, X. Xie, G. Zhang et al., "Exogenous phytoestrogenic molecule icaritin incorporated into a porous scaffold for enhancing bone defect repair," Journal of Orthopaedic Research, vol. 31, no. 1, pp. 164-172, 2013.

[31] Y. Yang, P. Chen, J. Wang, C. Lee, and J. Lai, "Prescription pattern of traditional Chinese medicine for climacteric women in Taiwan," Climacteric, vol. 12, no. 6, pp. 541-547, 2009.

[32] S. Kanai, N. Taniguchi, and H. Higashino, "Study of SokeiKakketu-To (Shu-Jing-Huo-Xue-Tang) in adjuvant arthritis rats," American Journal of Chinese Medicine, vol. 31, no. 6, pp. 879-884, 2003.

[33] Z. Tong, F. Yu, Z. Liu, and H. Liang, "Influence of ShuJinHuoXue tablets on ischemia reperfusion injury of animals' skeletal muscle," Molecules, vol. 17, no. 7, pp. 8494-8505, 2012.

[34] C. Chou, W. Wu, M. Chang, and D. Chang, "Efficacy and possible mechanisms of the chinese herbs suching-huo-hsueitang in the treatment of adjuvant-induced arthritis in rats," American Journal of Chinese Medicine, vol. 21, no. 2, pp. 159-170, 1993.

[35] H. Shu, H. Arita, M. Hayashida et al., "Anti-hypersensitivity effects of Shu-jing-huo-xue-tang, a Chinese herbal medicine, in CCI-neuropathic rats," Journal of Ethnopharmacology, vol. 131, no. 2, pp. 464-470, 2010.

[36] S. E. Jin, O. S. Kim, S.-R. Yoo et al., "Anti-inflammatory effect and action mechanisms of traditional herbal formula Gamisoyo-san in raw 264.7 macrophages," BMC Complementary and Alternative Medicine, vol. 16, no. 1, article no. 219, 2016.

[37] K. Wang, M. E. Coyle, S. Mansu, A. L. Zhang, and C. C. Xue, "Gentiana scabra Bunge. Formula for herpes zoster: biological actions of key herbs and systematic review of efficacy and safety," Phytotherapy Research, vol. 31, no. 3, pp. 375-386, 2017.

[38] F. Hinoshita, Y. Ogura, Y. Suzuki et al., "Effect of orally administered Shao-Yao-Gan-Cao-Tang (Shakuyaku-kanzo-to) on muscle cramps in maintenance hemodialysis patients: a preliminary study," American Journal of Chinese Medicine, vol. 31, pp. 445-453, 2003.

[39] P.-R. Yang, H.-F. Liang, Y.-H. Chu, P.-C. Chen, and Y.-Y. Lin, "Frequencies and prescription patterns of traditional Chinese medicine use among elderly patients in Taiwan: a populationbased study," Journal of Ethnopharmacology, vol. 169, pp. 328334, 2015.

[40] Y. Takao, Y. Takaoka, A. Sugano et al., "Shakuyaku-kanzoto (Shao-Yao-Gan-Cao-Tang) as treatment of painful muscle cramps in patients with lumbar spinal stenosis and its minimum effective dose," Kobe Journal of Medical Sciences, vol. 61, no. 5, pp. E132-E137, 2015.

[41] E. Rodríguez-Carballo, B. Gámez, and F. Ventura, "p38 MAPK signaling in osteoblast differentiation," Frontiers in Cell and Developmental Biology, vol. 4, p. 40, 2016.

[42] J. Huh, R. Ko, H. J. Jung, S. Y. Lee, and A. Asakura, "Glycogen synthase kinase $3 \beta$ promotes osteogenic differentiation of murine adipose-derived stromal cells," PLoS ONE, vol. 8, no. 1, Article ID e54551, 2013.

[43] C.-W. Chen, J. Sun, Y.-M. Li, P.-A. Shen, and Y.-Q. Chen, "Action mechanisms of Du-Huo-Ji-Sheng-Tang on cartilage degradation in a rabbit model of osteoarthritis," Evidence-Based Complementary and Alternative Medicine, vol. 2011, Article ID 571479, 7 pages, 2011.

[44] J.-Y. Wang, W.-M. Chen, C.-S. Wen, S.-C. Hung, P.-W. Chen, and J.-H. Chiu, "Du-Huo-Ji-Sheng-Tang and its active component Ligusticum chuanxiong promote osteogenic differentiation and decrease the aging process of human mesenchymal stem cells," Journal of Ethnopharmacology, vol. 198, pp. 64-72, 2017.

[45] S. Tan, X. Geng, J. Liu et al., "Xue-fu-Zhu-Yu decoction protects rats against retinal ischemia by downregulation of HIF- $1 \alpha$ and VEGF via inhibition of RBP2 and PKM2," BMC Complementary and Alternative Medicine, vol. 17, no. 1, p. 365, 2017.

[46] J. Lee, W. Hsu, T. Yen et al., "Traditional Chinese medicine, XueFu-Zhu-Yu decoction, potentiates tissue plasminogen activator against thromboembolic stroke in rats," Journal of Ethnopharmacology, vol. 134, no. 3, pp. 824-830, 2011.

[47] H.-H. Zhou, D.-L. Wu, L.-Y. Gao, Y. Fang, and W.-H. Ge, "L-tetrahydropalmatine alleviates mechanical hyperalgesia in models of chronic inflammatory and neuropathic pain in mice," NeuroReport, vol. 27, no. 7, pp. 476-480, 2016.

[48] Z. Guo, Y. Man, X. Wang et al., "Levo-tetrahydropalmatine attenuates oxaliplatin-induced mechanical hyperalgesia in mice," Scientific Reports, vol. 4, article 3905, 2014.

[49] H. Chu, G. Jin, E. Friedman, and X. Zhen, "Recent development in studies of tetrahydroprotoberberines: mechanism in antinociception and drug addiction," Cellular and Molecular Neurobiology, vol. 28, no. 4, pp. 491-499, 2008.

[50] Y. Zhang, C. Wang, L. Wang et al., "A novel analgesic isolated from a traditional Chinese medicine," Current Biology, vol. 24, no. 2, pp. 117-123, 2014.

[51] X. Zhou, W. Siu, C. Zhang et al., "Whole extracts of Radix Achyranthis Bidentatae and Radix Cyathulae promote angiogenesis in human umbilical vein endothelial cells in vitro and in zebrafish in vivo," Experimental and Therapeutic Medicine, vol. 13, no. 3, pp. 1032-1038, 2017.

[52] X. Kong, R. Wang, N. Tian, L. Li, N. Lin, and W. Chen, "Effect of Huogu II Formula (II) with medicinal guide radix achyranthis bidentatae on bone marrow stem cells directional homing to necrosis area after osteonecrosis of the femoral head in rabbit," Chinese Journal of Integrative Medicine, vol. 18, no. 10, pp. 761768, 2012.

[53] J. Chen, J. Deng, Y. Zhang et al., "Lipid-lowering effects of Danhong injection on hyperlipidemia rats," Journal of Ethnopharmacology, vol. 154, no. 2, pp. 437-442, 2014.

[54] G. Fan, X. Gao, H. Wang et al., "The anti-inflammatory activities of Tanshinone IIA, an active component of TCM, are mediated by estrogen receptor activation and inhibition of iNOS," The Journal of Steroid Biochemistry and Molecular Biology, vol. 113, no. 3-5, pp. 275-280, 2009.

[55] G. Yang, H. Zhang, R. Lin et al., "Down-regulation of CD40 gene expression and inhibition of apoptosis with danshensu in endothelial cells," Basic \& Clinical Pharmacology \& Toxicology, vol. 104, no. 2, pp. 87-92, 2009.

[56] M. N. Asl and H. Hosseinzadeh, "Review of pharmacological effects of Glycyrrhiza sp. and its bioactive compounds," Phytotherapy Research, vol. 22, no. 6, pp. 709-724, 2008.

[57] R. A. Isbrucker and G. A. Burdock, "Risk and safety assessment on the consumption of Licorice root (Glycyrrhiza sp.), its extract and powder as a food ingredient, with emphasis on the pharmacology and toxicology of glycyrrhizin," Regulatory Toxicology and Pharmacology, vol. 46, no. 3, pp. 167-192, 2006. 


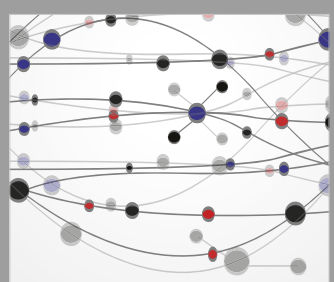

The Scientific World Journal
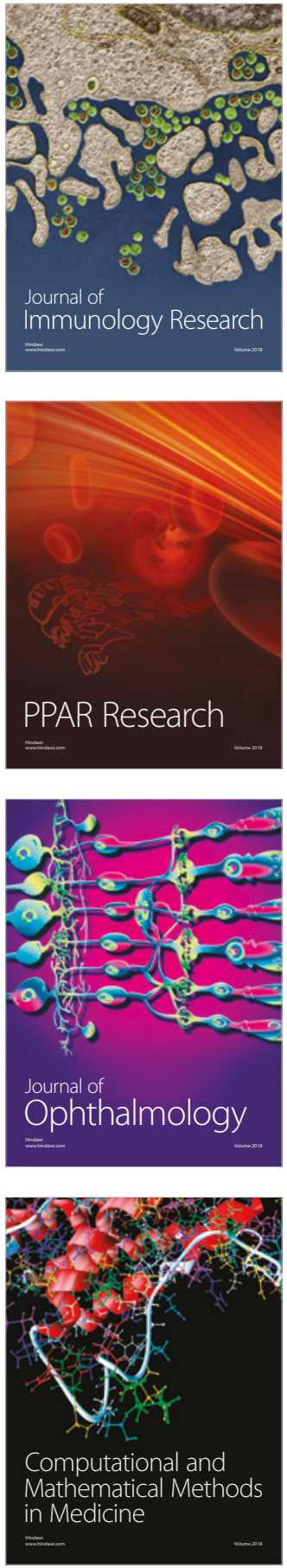

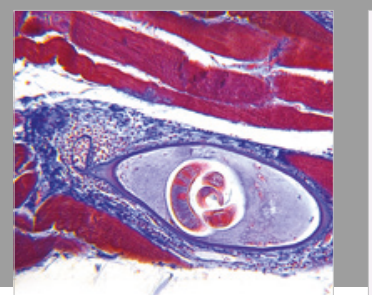

Gastroenterology Research and Practice

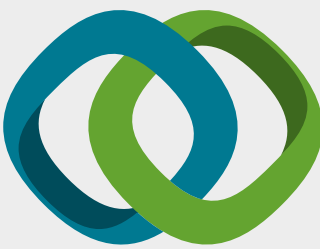

\section{Hindawi}

Submit your manuscripts at

www.hindawi.com
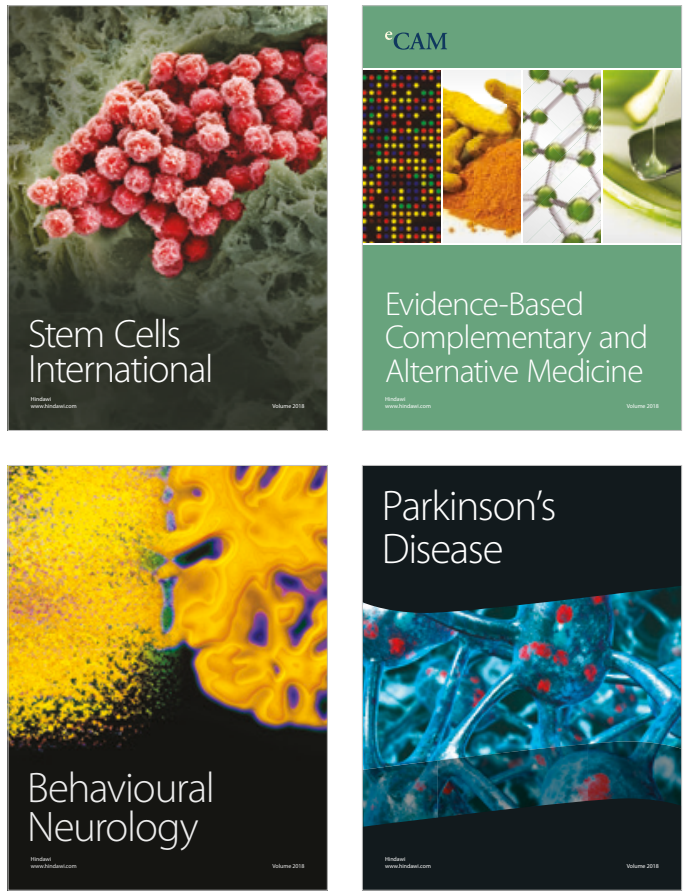

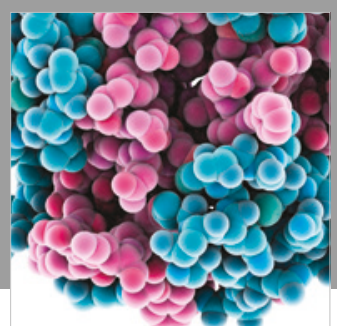

ournal of

Diabetes Research

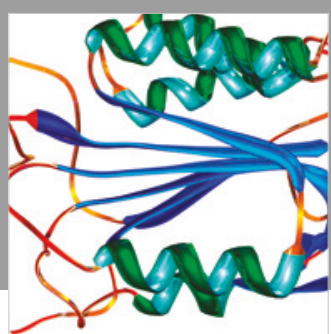

Disease Markers
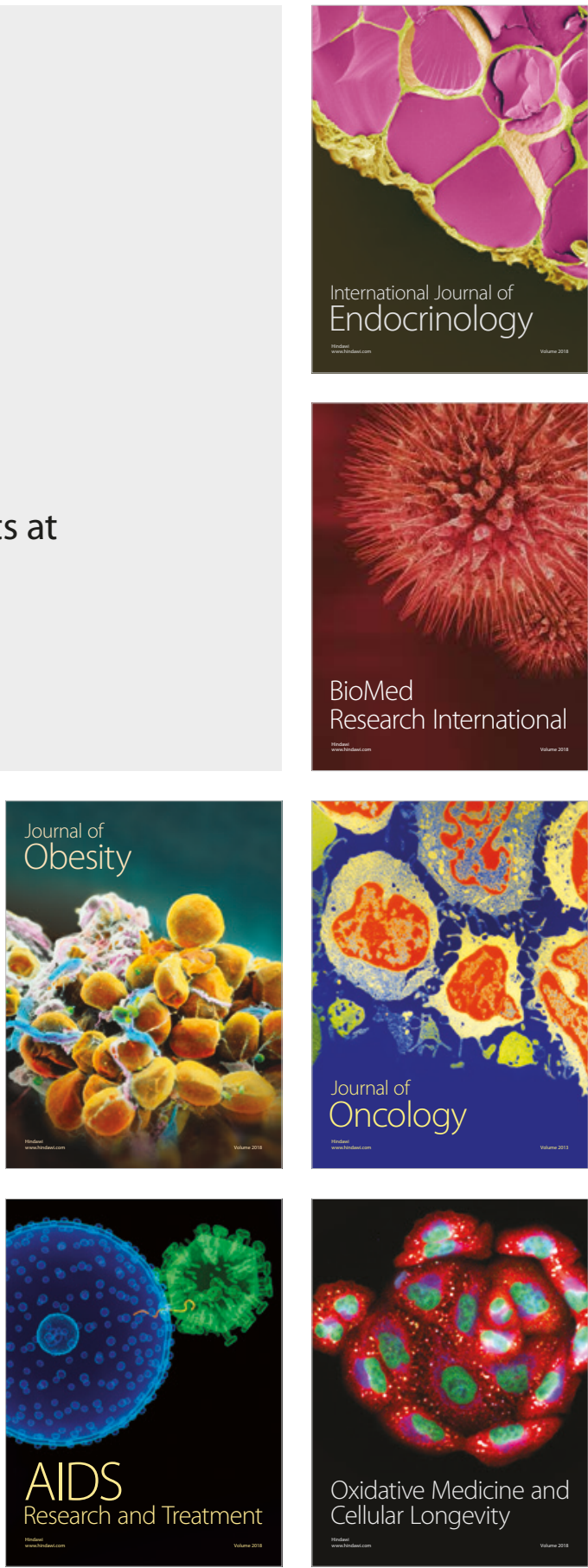